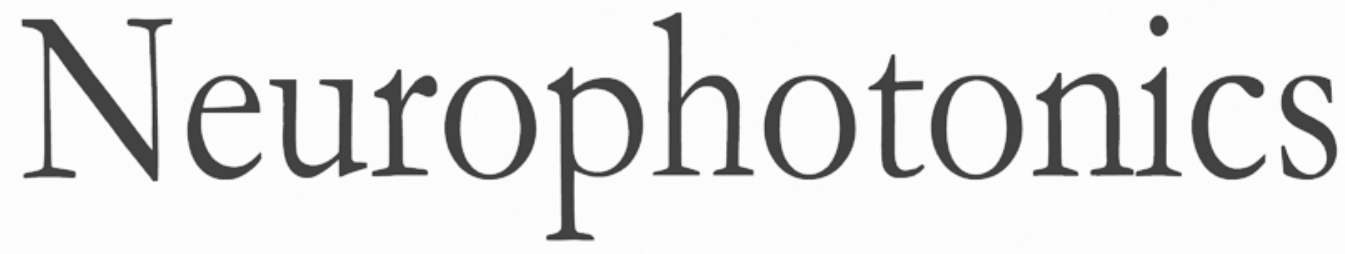

\title{
Separability of calcium slow waves and functional connectivity during wake, sleep, and anesthesia
}

Lindsey M. Brier

Eric C. Landsness

Abraham Z. Snyder

Patrick W. Wright

Grant A. Baxter

Adam Q. Bauer

Jin-Moo Lee

Joseph P. Culver 


\title{
Separability of calcium slow waves and functional connectivity during wake, sleep, and anesthesia
}

\author{
Lindsey M. Brier, ${ }^{a, *, \dagger}$ Eric C. Landsness, ${ }^{b, \dagger}$ Abraham Z. Snyder, ${ }^{a, b}$ Patrick W. Wright, ${ }^{c}$ Grant A. Baxter, ${ }^{a}$ \\ Adam Q. Bauer, ${ }^{a, c}$ Jin-Moo Lee, ${ }^{b}$ and Joseph P. Culver ${ }^{a, c, d}$ \\ aWashington University School of Medicine, Department of Radiology, St. Louis, Missouri, United States \\ bWashington University School of Medicine, Department of Neurology, St. Louis, Missouri, United States \\ 'Washington University in St. Louis, Department of Biomedical Engineering, St. Louis, Missouri, United States \\ dWashington University in St. Louis, Department of Physics, St. Louis, Missouri, United States
}

\begin{abstract}
Modulation of brain state, e.g., by anesthesia, alters the correlation structure of spontaneous activity, especially in the delta band. This effect has largely been attributed to the $\sim 1 \mathrm{~Hz}$ slow oscillation that is characteristic of anesthesia and nonrapid eye movement (NREM) sleep. However, the effect of the slow oscillation on correlation structures and the spectral content of spontaneous activity across brain states (including NREM) has not been comprehensively examined. Further, discrepancies between activity dynamics observed with hemoglobin versus calcium (GCaMP6) imaging have not been reconciled. Lastly, whether the slow oscillation replaces functional connectivity (FC) patterns typical of the alert state, or superimposes on them, remains unclear. Here, we use wide-field calcium imaging to study spontaneous cortical activity in awake, anesthetized, and naturally sleeping mice. We find modest brain state-dependent changes in infraslow correlations but larger changes in GCaMP6 delta correlations. Principal component analysis of GCaMP6 sleep/anesthesia data in the delta band revealed that the slow oscillation is largely confined to the first three components. Removal of these components revealed a correlation structure strikingly similar to that observed during wake. These results indicate that, during NREM sleep/anesthesia, the slow oscillation superimposes onto a canonical FC architecture. $\odot$ The Authors. Published by SPIE under a Creative Commons Attribution 4.0 Unported License. Distribution or reproduction of this work in whole or in part requires full attribution of the original publication, including its DOI. [DOI: 10.1117/1.NPh.6.3.035002]
\end{abstract}

Keywords: calcium; functional connectivity; hemoglobin; sleep; slow oscillation.

Paper 19016R received Mar. 1, 2019; accepted for publication Jun. 12, 2019; published online Jul. 13, 2019.

\section{Introduction}

Correlation analysis of spontaneous brain activity has been increasingly used in systems neuroscience. ${ }^{1-4}$ This methodology provides a powerful approach for mapping various cognitive processes as well as understanding specific clinical populations. ${ }^{5}$ Specifically, functional connectivity (FC) analysis maps co-ordinate brain activity at rest in a remarkably reproducible manner. ${ }^{6}$ Canonically, FC analysis is performed on data filtered in the infraslow band, that is, $0.009-0.08 \mathrm{~Hz}{ }^{7}$ In addition, brain activity used in this type of analysis is typically derived from hemodynamic fluctuations calculated by using imaging modalities such as functional magnetic resonance imaging $(\mathrm{fMRI})^{8}$ or diffuse optical tomography ${ }^{9}$ in humans. Alternatively, wide-field optical imaging modalities, such as optical intrinsic signal (OIS) imaging, allow for the same FC analysis to be performed in mice. ${ }^{10}$ More recently, FC patterns extracted from wide-field optical imaging have been identified within neural calcium dynamics in mice that express genetically encoded calcium indicators (GECIs). ${ }^{11-13}$ These fluorophores have extended the temporal frequency range in which FC can be studied and provide detailed maps of FC across the whole cortex. ${ }^{14}$ Another advantage of fluorophore imaging is that fluorescence is dependent on cell-specific calcium flux, providing a more direct read-out of neural activity that is independent of neurovascular coupling. However, recording fluorescence fluctuations in higher

\footnotetext{
*Address all correspondence to Lindsey M. Brier, E-mail: brierl@wustl.edu
}

tThese authors contributed equally to this work. frequency bands, such as the delta band (historically $0.4-4.0 \mathrm{~Hz}$ ), introduces potential confounds. In particular, the delta band "slow oscillation" that is a hallmark of nonrapid eye movement (NREM) sleep appears to be a distinct physiological phenomenon from resting state $\mathrm{FC}$, and it will be important to understand the potential confounding effects of these slow oscillations on FC analysis.

The "slow oscillation" (alternatively, up and down states) refers to a particular electrophysiological phenomenon in the delta band first described in anesthetized cats by Steriade et al. ${ }^{15}$ The defining feature is quasiperiodic (nominally, 0.5-1.5 Hz) high amplitude fluctuations of neural excitability synchronously affecting the entire cerebral cortex. The slow oscillation is a defining feature of natural slow-wave sleep and plays a crucial role in memory consolidation. ${ }^{16}$ The slow oscillation is also present under some forms of anesthesia. ${ }^{17}$ As the mouse functional brain imaging literature expands into higher frequencies (e.g., the delta band), it will be imperative to understand the effect that different brain states (wake versus anesthesia versus natural sleep) have upon correlation structures since the traditional correlation analysis used in FC mapping may be confounded by the presence of large amplitude delta-band activity.

Resting-state fMRI studies in humans have demonstrated reliable FC changes during sleep and anesthesia compared to wake in the infraslow frequency band, which does not encompass slow oscillation dynamics. ${ }^{18-22}$ Utilizing the higher temporal resolution of GECIs, we have previously shown much larger changes in delta band correlation structures due to ketamine/ xylazine anesthesia compared to wake. ${ }^{13}$ Other groups have suggested that the slow oscillation has a significant impact on delta 
band correlation structures, particularly driven by the globally synchronous high amplitude slow oscillation propagations. ${ }^{23-25}$ Specifically, delta band FC during ketamine/xylazine anesthesia produces a nearly binary pattern with large regions of correlations and anticorrelations. By contrast, delta band FC during wake shows more focal homotopic connectivity patterns. ${ }^{13}$ No comparison currently exists for delta band FC during any of the stages of natural sleep in the GECI literature. Therefore, the comparative effects of the slow oscillation on correlation structures, induced either spontaneously during natural sleep or mechanistically by anesthesia, have not been evaluated. It is not clear how correlation structures compare across brain states, frequency bands, or calcium/hemoglobin dynamics analyzed in the mouse. Finally, it is not clear to what extent the slow oscillation is a distinct phenomenon, i.e., whether it replaces or superimposes on top of underlying wake-like spontaneous delta activity.

Herein, we exploit genetically engineered mice with the calcium indicator GCaMP6 driven by the Thyl promoter in excitatory neurons ${ }^{13}$ to directly compare wide-field correlation structures of hemoglobin and calcium signals in both the infraslow and delta bands during wakefulness, two types of anesthesia, and NREM sleep. Additionally, we evaluate the separability of the slow oscillation from spontaneous wake-like FC patterns. To rigorously address these questions, we have concurrently acquired GECI/OIS imaging of calcium and hemoglobin dynamics together with electroencephalography/ electromyography (EEG/EMG) to enable accurate assessment of arousal state. We use a linear decomposition analysis, specifically, principal component analysis (PCA), to separate the slow oscillation from ongoing intrinsic activity to determine whether the slow oscillation replaces wake-like intrinsic delta activity during sleep/anesthesia or rather superimposes on it.

\section{Materials and Methods}

\subsection{Animals}

A total of six, three-month-old transgenic GCaMP6 Thyl/ C57BL6 male mice (JAX strain: C57BL/6J-Tg(Thy1GCaMP6f)GP5.5Dkim/J; stock: 024276) were used in the present study.

\subsection{Surgery}

Prior to data collection, the mice underwent surgical implantation of two stainless steel lateral EEG bone screws located at $-1 \mathrm{~mm}$ posterior to bregma, and $\pm 5 \mathrm{~mm}$ lateral to bregma, a cerebellum bone screw to act as reference, and an EMG wire placed in the neck [Fig. 1(a)]. Using previously described methods, ${ }^{26}$ a Plexiglass head cap was then fixed with a translucent (a)

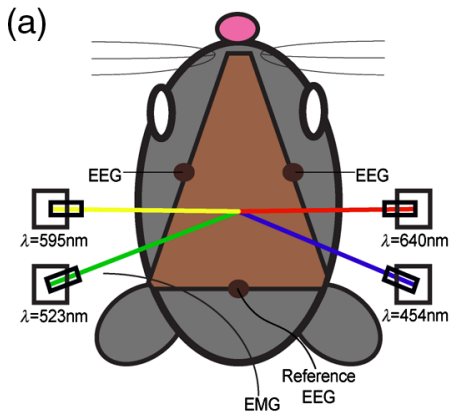

(b)

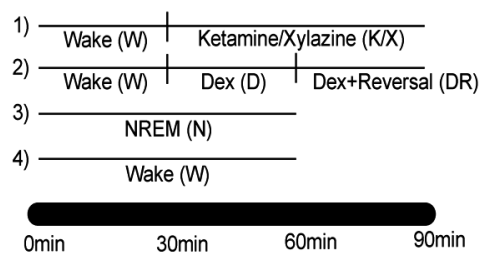

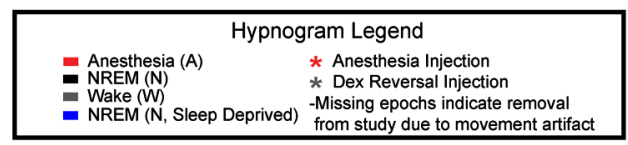
-Missing epochs indicate removal
from study due to movement artifact

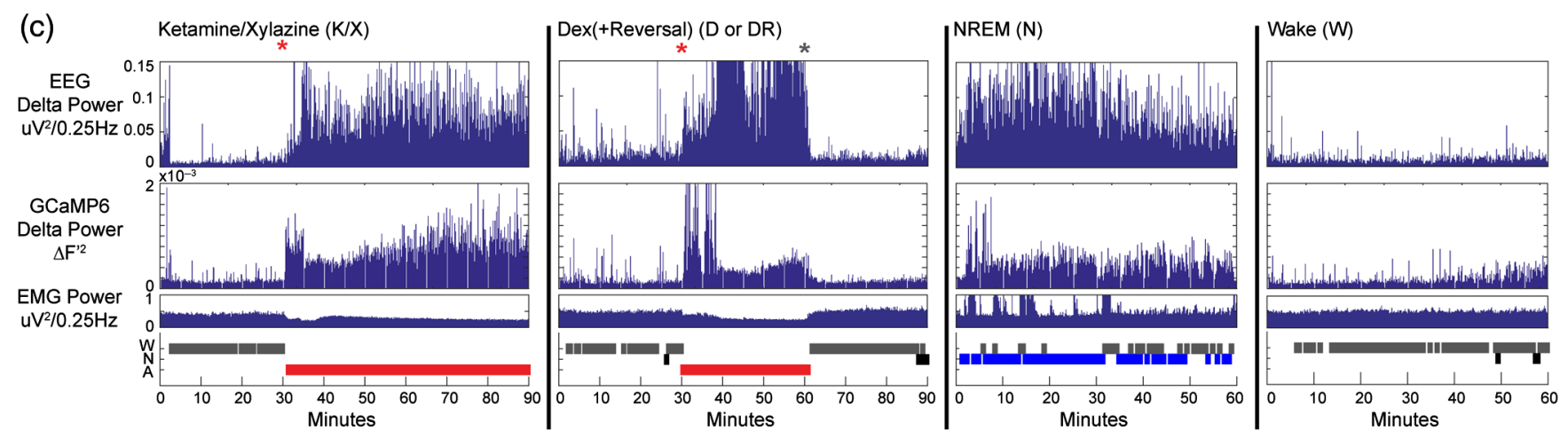

Fig. 1 Concurrent GCaMP6 fluorescence, OIS imaging, and EEG acquisition system with experimental design. (a) Mouse head schematic of chronically implanted Plexiglass window used for consistent, repeated imaging experiments (brown). Two EEG screws were implanted bilaterally in the skull, with a reference EEG electrode near the cerebellum and EMG wire threaded through the neck as shown. OIS imaging was performed using sequential LED illumination supplied by three LEDs $(523,595$, and $640 \mathrm{~nm}$ ). GCaMP6 fluorescence imaging was performed using a $454 \mathrm{~nm}$ LED for excitation. (b) Outline of experimental design. For experiments (1) and (2), $30 \mathrm{~min}$ of spontaneous wake data was acquired, injection of the anesthetic followed, with the reversal agent given in run (2) at $60 \mathrm{~min}$ to reverse the effects of dex. In runs (3) and (4), 60 min of spontaneous NREM (if sleep deprived) or wake (if not sleep deprived) data was acquired to be scored. (c) Example delta $(0.7-3.0 \mathrm{~Hz})$ power time course for EEG and GCaMP6 data with corresponding EMG and hypnogram for one mouse during ketamine/xylazine (K/X), dex (D), dex+reversal (DR), NREM (N), and wake (W). 
adhesive cement (C\&B-Metabond, Parkell Inc., Edgewood, New York) to allow for chronic, repeated imaging. Animals were allowed 1 week to recover and housed in group cages on 12-h/12-h light/dark cycles with lights on at 6:00 A.M. All studies were approved by the Washington University School of Medicine Animal Studies Committee and followed the guidelines of the National Institutes of Health's Guide for the Care and Use of Laboratory Animals.

\subsection{Anesthesia}

Animals were serially imaged under two different anesthetic agents: ketamine/xylazine $(86.9 \mathrm{mg} / \mathrm{kg}$ ketamine and $13.4 \mathrm{mg} / \mathrm{kg}$ xylazine) and dexmedetomidine ("dex" $0.5 \mu \mathrm{g} / \mathrm{g}$ ). These anesthetics are, respectively, an NMDA receptor antagonist (ketamine) ${ }^{27}$ and an alpha-2 adrenergic agonist (xylazine, dex). ${ }^{28}$ These specific anesthetics were selected to obtain findings across commonly used anesthetics in mice (ketamine/ xylazine $)^{29}$ and humans (dex). ${ }^{30,31}$ Although slow oscillations have been reported after ketamine alone, ${ }^{17,32}$ xylazine is commonly adjunctively used in rodent experiments to maximally synergize analgesia, immobility, muscle relaxation, and sedation. ${ }^{33,34}$ The anesthetic experiments consisted of $30 \mathrm{~min}$ of recording spontaneous wake followed by intraperitoneal injection of the anesthetic agent and recording for a subsequent $60 \mathrm{~min}$ [Fig. 1(b)]. In the case of dex, after $60 \mathrm{~min}$ of recording, the reversal agent, atipamezole, at a dosing of $0.5 \mu \mathrm{g} / \mathrm{g}$ at $50 \mu \mathrm{g} / \mathrm{ml}$, was given. Between all recording sessions, there was a minimum of 2-4 days to allow for sufficient washout of anesthetic agents and the order of the agents administered was randomized.

\subsection{Sleep Deprivation}

After a washout period of 2-4 days post-anesthetic use, the second part of the experiment consisted of $6 \mathrm{~h}$ of sleep deprivation starting at lights-on followed by an hour of continuous recording. Sleep deprivation was necessary to increase the amount of NREM data that could be collected during an hour imaging session with a head-fixed animal and was performed by placing the mice in an unfamiliar, enriched environment, as previously reported. ${ }^{35}$ If the animal was noted to be behaviorally sleeping (immobile, eyes closed), unfamiliar objects and bedding material were placed in their cage. Alternatively, objects were moved to different locations within the cage or puffs of air were directed at the immobile animals while direct handling was kept to a minimum. Animals were not disturbed while actively awake (feeding, moving, and grooming).

\subsection{Awake Imaging}

After 1-4 days post-sleep deprivation, the mice were imaged for 60 min of spontaneous wake data for baseline comparison.

\subsection{Imaging}

As previously described, ${ }^{13}$ animals were placed in a black, felt pouch with their heads secured in place under the LEDs [Fig. 1(a)]. An overhead camera was used for imaging. Mice were acclimatized to the apparatus the week prior to data collection. Sequential illumination was provided by four LEDs: $454 \mathrm{~nm}$ (GCaMP6 excitation), $523 \mathrm{~nm}, 595 \mathrm{~nm}$, and $640 \mathrm{~nm}$ (Mightex Systems, Pleasanton, California). Images were acquired with a cooled, frame-transfer EMCCD camera (iXon
897, Andor Technologies, Belfast, Northern Ireland, United Kingdom) in combination with an $85 \mathrm{~mm} \mathrm{f} / 1.4$ camera lens (Rokinon, New York, New York) at a frame rate of $16.81 \mathrm{~Hz} /$ LED channel. Allowing for a modest buffer beyond the Nyquist frequency of $8.4 \mathrm{~Hz}$, this set-up allowed for calcium data analysis at frequencies up to $6 \mathrm{~Hz}$. The field-of-view covered most of the convexity of the cerebral cortex with anterior-posterior coverage from the olfactory bulb to the superior colliculus. The resulting pixel resolution was $\sim 78 \mu \mathrm{m}^{2}$. For each mouse, the recording duration was between 60 and 90 min per brain state. All imaging data were acquired in 5-min imaging "runs."

\subsection{EEG Data Analysis}

On the day of recording, the EEG screws and EMG wire were connected to an amplifier and data were collected at $10,000 \mathrm{~Hz}$ (Power Lab EEG Amplifier, AD Instruments, Dunedin, New Zealand). This data was then downsampled to $256 \mathrm{~Hz}$ offline. The authors (L.M.B. and E.C.L.) scored the EEG and EMG data in 10 -s epochs according to standard criteria ${ }^{36}$ as either wake, sleep, anesthesia, or movement artifact. The EEG data were low-pass filtered at $40 \mathrm{~Hz}$ to remove artifacts primarily associated with motor activity, then a 10-s Hann window was applied to each epoch. Finally, the fast Fourier transform (FFT) was computed and squared to obtain the EEG power.

\subsection{Image Processing}

GCaMP6/hemoglobin images underwent image processing, as described elsewhere, ${ }^{13,37}$ and summarized here. A binary brain mask was created and applied by tracing along with the fieldof-view framed by the scalp retraction procedure using the roipoly.m procedure in MATLAB. After first subtracting the ambient light levels, temporal detrending was performed by fitting the data with a fourth order polynomial that was subsequently regressed out of the time series for each pixel. Spatial detrending was performed by pixelwise averaging time traces to generate a regressor for the time series data. The fluorescence and 523-nm reflectance data were mean normalized and the ratio of the fluorescence emission data divided by the $523 \mathrm{~nm}$ reflectance data was used to correct the fluorescence data for absorption dynamics due to oxygenated-hemoglobin $\left(\mathrm{HbO}_{2}\right)$ and deoxygenated-hemoglobin (HbR) dynamics. The modified Beer-Lambert's law was solved using the 523-, 595-, and 640$\mathrm{nm}$ wavelength reflected intensities to yield oxygenated- and deoxygenated-hemoglobin fluctuations. Images were smoothed with a $5 \times 5$ Gaussian filter. The time traces for all pixels within the brain mask were averaged to compute a global signal, which then was removed by regression from every pixel's individual time trace to eliminate globally shared variance. Power spectral analysis of the GCaMP6 and $\mathrm{HbO}_{2}$ signals was computed with a Hann window and FFT. For zero-lag correlation computations, a Butterworth bandpass filter of 0.009 to $0.08 \mathrm{~Hz}$ or 0.7 to $3.0 \mathrm{~Hz}$ was applied to the data and seed-based zero-lag Pearson correlation coefficients were calculated per pixel using prespecified seed locations. The $0.009-0.08 \mathrm{~Hz}$ band was chosen to replicate infraslow analysis typically done in fMRI. ${ }^{38}$ The $0.7-3.0 \mathrm{~Hz}$ band encompasses the three instantiations of the slow oscillation produced by NREM sleep and the two anesthetics used in this study [Fig. 2(a)]. In the following, we refer to this frequency range as "delta," which differs somewhat from the conventional definition of "delta" used in human EEG $(0.4-4.0 \mathrm{~Hz}){ }^{39}$ Seeds corresponded to the left cingulate, motor, somatosensory, 
(a)

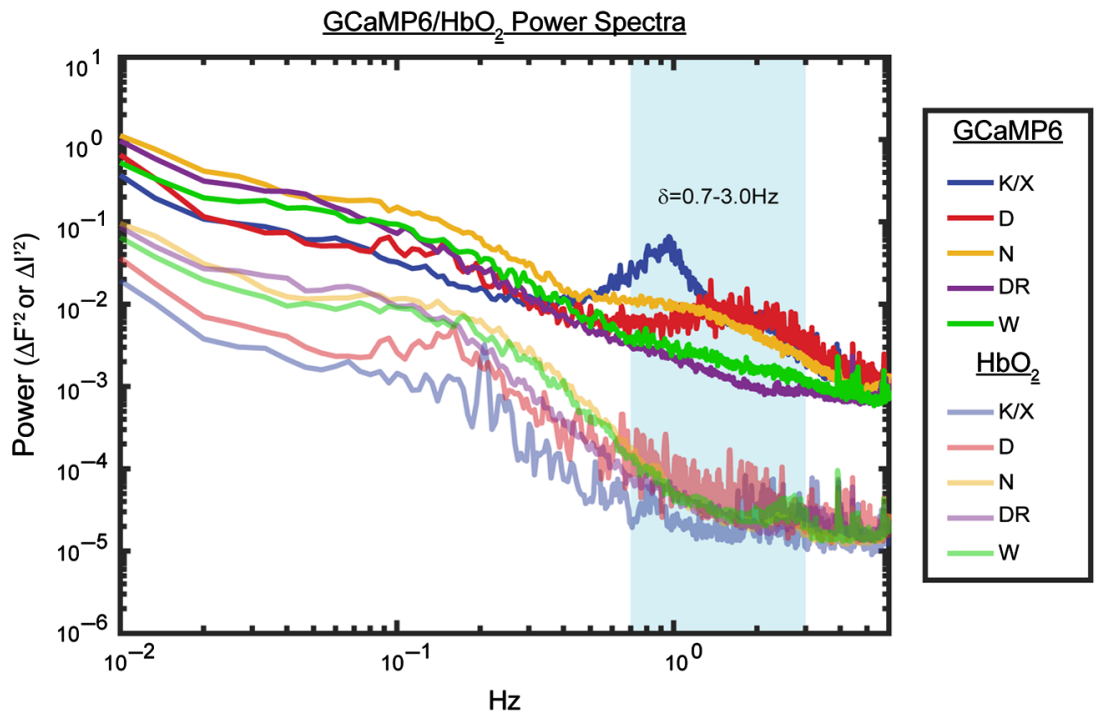

(b)
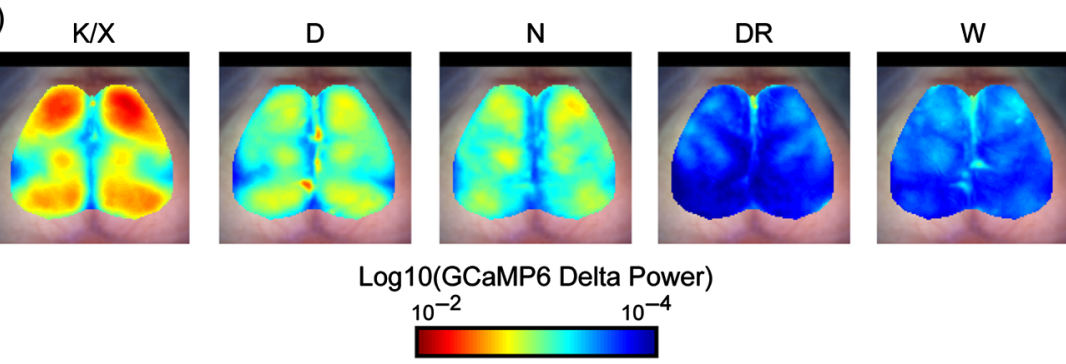

(c)
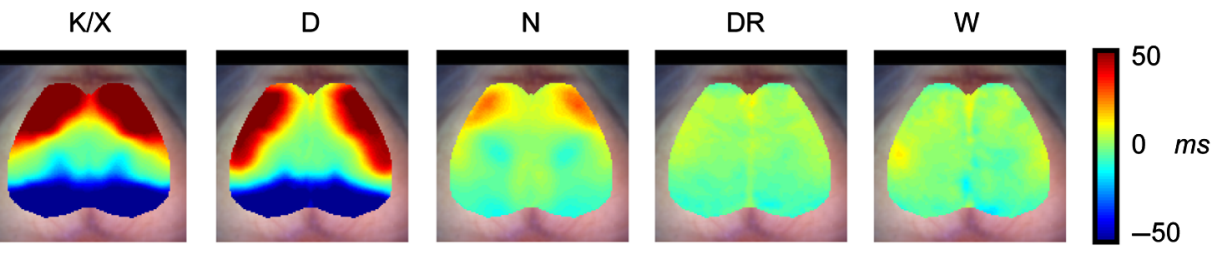

GCaMP6 Lags, $0.7-3 \mathrm{~Hz}$

Fig. 2 Spectral densities in anesthesia, NREM, and wake. (a) The average $(n=5)$ GCaMP6 (solid lines) and $\mathrm{HbO}_{2}$ (translucent lines) power for each brain state plotted with the delta band $(0.7-3.0 \mathrm{~Hz}$ ) highlighted. (b) Log10 average $(n=5)$ GCaMP6 delta $(0.7-3.0 \mathrm{~Hz})$ power topographs for each brain state. (c) Average $(n=5)$ GCaMP6 delta lag topographies for each brain state.

retrosplenial, auditory, visual, and parietal cortices and were selected to represent the major cortical regions (defined by function) within the field-of-view of the imaging system. The specific cortical location within each region was based-off of previously published canonical seed locations in the Paxinos atlas space and was slightly modified (frontal and olfactory seeds were excluded because of the slightly more condensed field-of-view presented here). ${ }^{10,13}$ A Fisher z-transform was applied to the correlation coefficients before averaging and performing statistics (see below). The z-transformed averaged data were then reverse transformed to correlation coefficients.

We used lag analysis to capture the propagation properties of delta activity considered as an average. Specifically, the data were reprocessed without global brain signal regression. Lagged cross-correlations were computed for single-pixel traces versus the global brain signal. The correlation max was found as in Wright et al., ${ }^{13}$ and the corresponding shift by cubic spline interpolation (in ms) was defined as voxelwise lag. In addition, cross-correlation analysis between the delta EEG and GCaMP6 PCA data was performed. The max pixelwise correlation was plotted as an image as well as the lag shift necessary to produce the max correlation.

\subsection{Artifact Rejection}

During the EEG/EMG scoring, any fluctuations in EMG signal were scored as "artifact" and the corresponding EEG/GCaMP6/ hemoglobin data were discarded. A binary spatial mask, as described above, was applied to all analyses to account for imaging artifact generated by nonbrain regions.

\subsection{Statistics}

A total of six, three-month-old transgenic GCaMP6 Thyl/ C57BL6 male mice (JAX Strain: C57BL/6J-Tg(Thy1GCaMP6f)GP5.5Dkim/J; stock: 024276) were used in the present study. The intent was to study five mice in each brain state. However, owing to one mortality (likely due to multiple anesthetic administrations), one mouse in the original fivemouse cohort died and was replaced by a sixth mouse. Supplementary Figure S2 lists which mouse was studied in each 
of the experimental states. With the mix of paired and independent data-points collected here, there is no established method to exchange condition labels within the dataset to justify using a permutated linear mixed model approach and no other mixed model approach has been proposed or validated for this purpose. Therefore, to quantify our contention regarding brain state-dependent correlation patterns and that post-principal component removal (see below) ketamine/xylazine, dex, and NREM functional patterns resemble wake FC, a random effects statistic ${ }^{40}$ at each pixel (mean/std) was calculated. Briefly, random effects analysis attempts to predict an outcome based on a linear combination of multiple variables that explains the heterogeneity within a sample. Here, we use this type of analysis to locate pixels that cross a predetermined threshold considering all the mice used for each brain state. Pixels at which the correlation strength exceeded the predetermined threshold were considered statistically significant. Binary maps indicating functional connections significantly greater than 0 (using a threshold for pixels with a random effects statistic greater than 2.58) were created. The regions specific to each modulated brain state (anesthesia/sleep) and wake were quantified separately by summing uniquely significant pixels in each case to evaluate whether FC patterns were consistent across brain state.

\subsection{Principal Component Analysis}

Singular value decomposition analysis was computed on all of the $0.7-3.0 \mathrm{~Hz}$ filtered GCaMP6 data using MATLAB to remove the first three principal components (PCs) and analyze them independently. Representations of PCs were computed by normalizing the $\Delta F / F$ signal to a 0 to 1 scale and then averaging across mice. The amount of variance each PC represented was computed.

\section{Results}

\subsection{Optical System with Simultaneous EEG to Accurately Classify Brain State}

As described elsewhere, ${ }^{13,41}$ we evaluated correlation patterns during wake and anesthesia using wide-field, whole dorsal cortex (olfactory bulb to superior colliculus) optical imaging. To examine the influence of brain state on correlation structures, EEG/EMG recordings were simultaneously collected for scoring specific time epochs as wake, NREM sleep, anesthesia, or movement artifact [Fig. 1(a)]. The experimental protocol involved recording calcium and hemoglobin dynamics across the cortex during wake (W), natural NREM sleep (N), and two different anesthetized states: ketamine/xylazine $(\mathrm{K} / \mathrm{X})$ and dexmedetomidine (“dex," D) [Fig. 1(b)]. Ketamine/xylazine and dex are known to induce slow oscillations similar to those observed during NREM sleep. ${ }^{42,43}$ Dex has the added advantage of being reversible with atipamezole. ${ }^{31,44}$

To classify brain state, EEG and EMG were scored in 10-s epochs by standard criteria ${ }^{36}$ as either wake, NREM sleep, anesthesia, or artifact and visualized using hypnograms [Fig. 1(c); see Supplementary Figure S1 for quantification of time spent in each brain state]. Delta power analysis of the EEG and GCaMP6 signals revealed increases in delta power aligned with transition of brain state score, indicating NREM sleep/ anesthesia and relative decreases in delta power aligned with wake [Fig. 1(c)]. As previously demonstrated, ${ }^{13}$ during sleep and anesthesia, there was an increase in the whole-brain GCaMP6 fluorescence power, mostly within the $0.7-3 \mathrm{~Hz}$ frequency band relative to wake or after dex reversal [Fig. 2(a)]. This narrower frequency band, relative to the more traditionally defined 0.4 $4 \mathrm{~Hz}$ delta band, was used for analysis in the present study going forward to focus on frequency content sensitive to the states in this study. The delta increase was specific to the GCaMP6 data under anesthesia and natural sleep. No detectable brain state-specific changes were present in the hemoglobin data, presumably due to limited hemoglobin dynamics at frequencies above $\sim 0.2 \mathrm{~Hz}^{45,46}$

Given the increase in the spatially averaged delta power, we investigated topographic differences in the power of the fluorescence signal in the $0.7-3 \mathrm{~Hz}$ range of the GCaMP6 data [Fig. 2(b)]. There was a significant increase in the delta power under anesthesia and during NREM sleep, and this increase was significantly modulated across distinct cortical regions, especially in motor, somatosensory, and visual areas relative to wake and dex reversal (power topography maps for each individual mouse are shown in Supplementary Figure S2). Further, as shown first using scalp EEG recordings ${ }^{47}$ and later calcium dynamics ${ }^{48}$ the dominant spatial property of the slow oscillation is the anterior to posterior propagation of the slow wave. This feature was captured in our GCaMP6 sleep/anesthesia data and displayed in real time (Fig. 6, Video 1, Supplementary Figure S3A). Using single-pixel cross-correlation analysis with the global brain signal, "lag" analysis captured a front-to-back topography across the anesthesia and natural sleep states, which was absent in wake [Fig. 2(c)]. Collectively, these results support the accurate classification of the different brain states and set the stage for further brain state-specific correlation analysis with the calcium (GCaMP6) and hemoglobin data.

\subsection{Hemoglobin and GCaMP6 Infraslow Correlation Structures Modestly Change Across Brain State, While GCaMP6 Delta Correlation Structures Appear More State-Dependent}

Given that our wide-field calcium spectral data show differences between wake and sleep/anesthesia in the delta band and that has largely been attributed to the presence of the slow oscillation, we then tested the hypothesis that hemoglobin and GCaMP6 correlation structures within the infraslow (0.009$0.08 \mathrm{~Hz}$ ) frequency range would not vary with brain state due to the slow oscillation being filtered out of the data. Seed-based, zero-lag correlational analysis with both the calcium and hemoglobin signal in the infraslow range showed the previously demonstrated $^{10}$ contralateral homotopic correlations and anticorrelations between functionally distinct regions [Figs. 3(a) and 3(b), top] i.e., "FC." To quantify spatial similarity across brain state and calcium/hemoglobin dynamics, we calculated significant topographic patterns using a random effects analysis across the five mice in each brain state. This analysis created binary maps representing areas of FC with correlation strength significantly greater than 0 [Figs. 3(a) and 3(b), middle]. The binary map for each brain state (besides wake) was then compared to the wake binary map and the overlap between the two compared brain states is shown in green. Also mapped are brain regions with significant correlation structures unique to either wake (white) or the anesthesia/sleep/dex reversal brain states (blue). The area unique to each modulated brain state (blue) or wake (white) [Figs. 3(a) and 3(b), bottom] made up, on average, less than $15 \%$ of the entire field-of-view in both the hemoglobin and GCaMP6 data. 

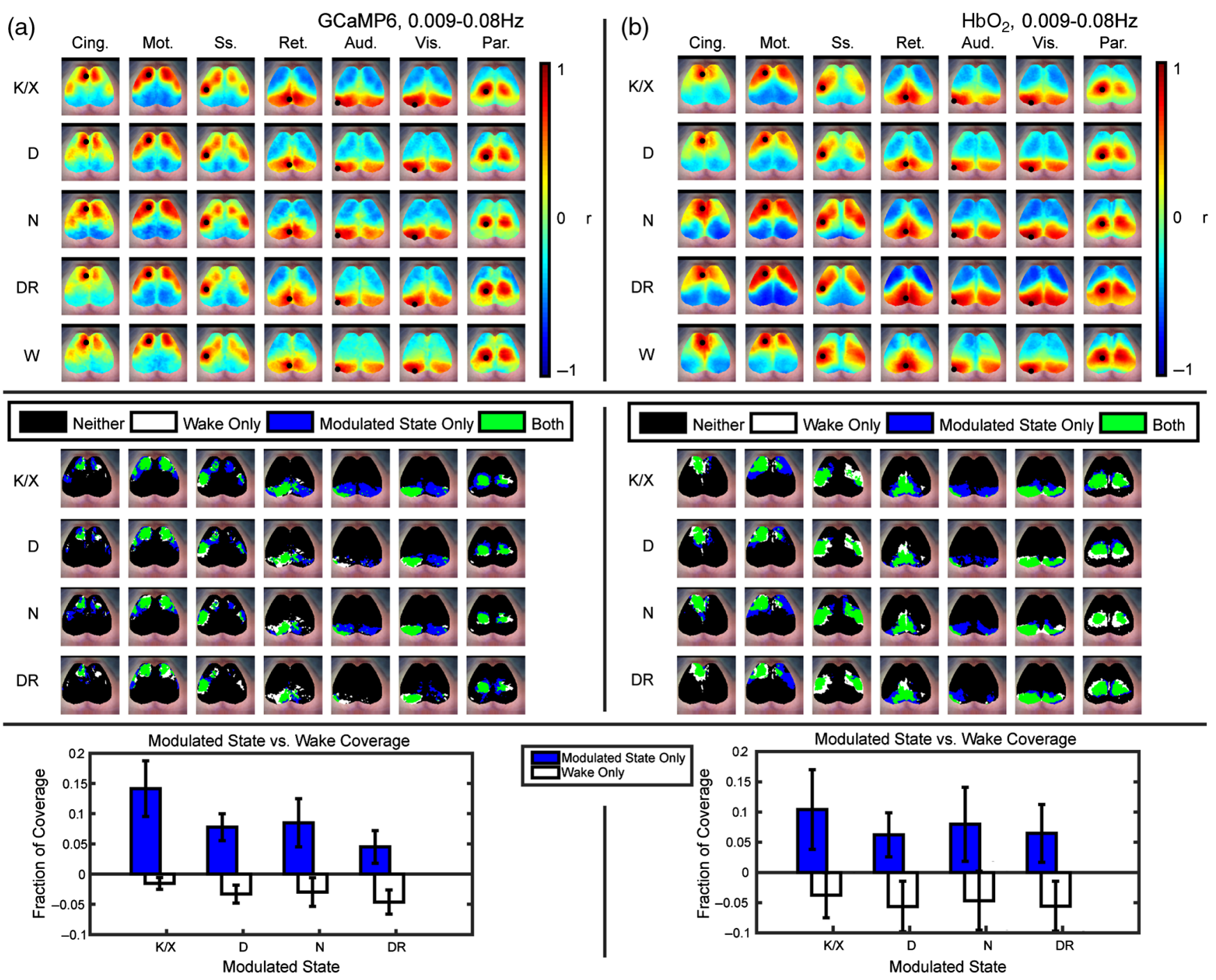

Fig. 3 Infraslow correlation structures for each brain state using hemoglobin or GCaMP6 dynamics. Top: Seed based zero-lag correlation maps $(n=5)$ across brain state calculated on (a) GCaMP6 or (b) oxygenated hemoglobin $\left(\mathrm{HbO}_{2}\right)$ data within the infraslow band. Middle: random effects overlap maps created by comparing each brain state to wake using (a) GCaMP6 or (b) oxygenated hemoglobin data. Bottom: fraction of significant pixels within the field-of-view unique to either the modulated brain state (blue) or wake state (white). Error bars are standard deviations across seeds.

Next, we performed the same seed-based correlation analysis in the delta range $(0.7-3.0 \mathrm{~Hz})$ on the GCaMP6 data, anticipating an exaggerated difference in FC across brain states due to the presence of the slow oscillation. Hemoglobin dynamics were not analyzed in this band as they did not demonstrate a peak in this spectral region. A highly symmetric topography marked by antiphase relations across anterior versus posterior areas was observed only in anesthesia and sleep [Fig. 4(a)]. By contrast, this pattern was absent in the wake state [Fig. 4(a), lowest row], which instead produced FC structures with contralateral homotopic correlations and anticorrelations between functionally distinct regions. These differences were confirmed with random effects analysis by creating binary maps and performing the same overlap calculations, as described previously [Fig. 5(a), top, and Fig. 5(b)]. The fraction of coverage unique to each modulated brain state was greater in the delta range relative to the infraslow range and was different between each modulated brain state.

\subsection{The Slow Oscillation is Superimposed on Delta Wake FC Patterns}

Given the differences in correlation structure within the delta range of the GCaMP6 data, we next investigated whether PCA could be used to separate or remove the delta spatiotemporal feature (the slow oscillation) present during sleep and anesthesia from other spontaneous brain activity. We hypothesized that the removal of the slow oscillation would reveal FC maps similar to the delta GCaMP6 wake maps, specifically, homotopic correlations and anticorrelations between functionally distinct regions. PCA on the delta GCaMP6 data revealed striking differences in the first three PCs contrasting wake versus NREM and anesthesia [Fig. 4(b)]. Specifically, the summation of PC1, PC2, and PC3 captured the major front-to-back feature of the slow oscillation during NREM and anesthesia (Fig. 7, Video 2). Compared to previously reported correlation coefficients between GCaMP6 and EEG, ${ }^{49}$ the cross-correlation 
(a)

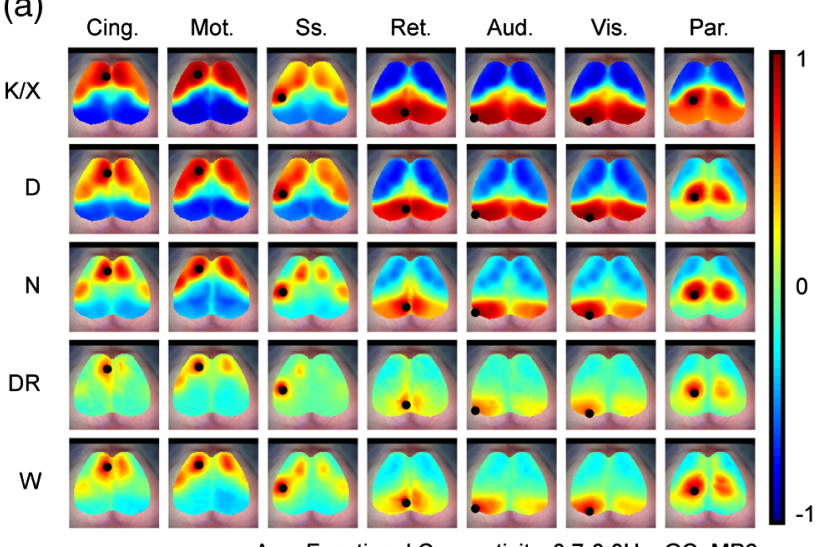

Avg. Functional Connectivity, 0.7-3.0Hz, GCaMP6

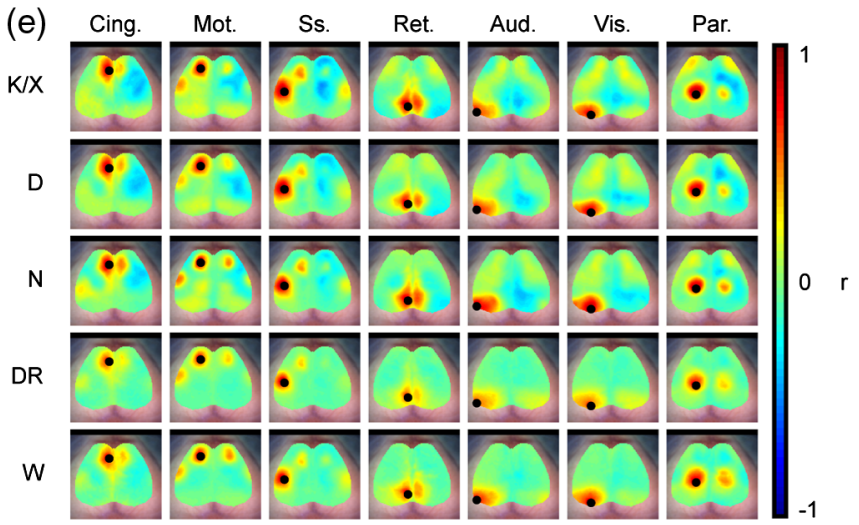

Avg. Functional Connectivity, PC1-3 Removed, 0.7-3.0Hz, GCaMP6 (b)

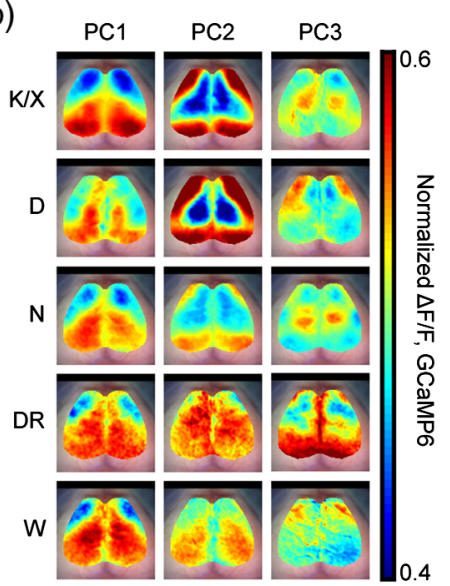

(c)

(d)

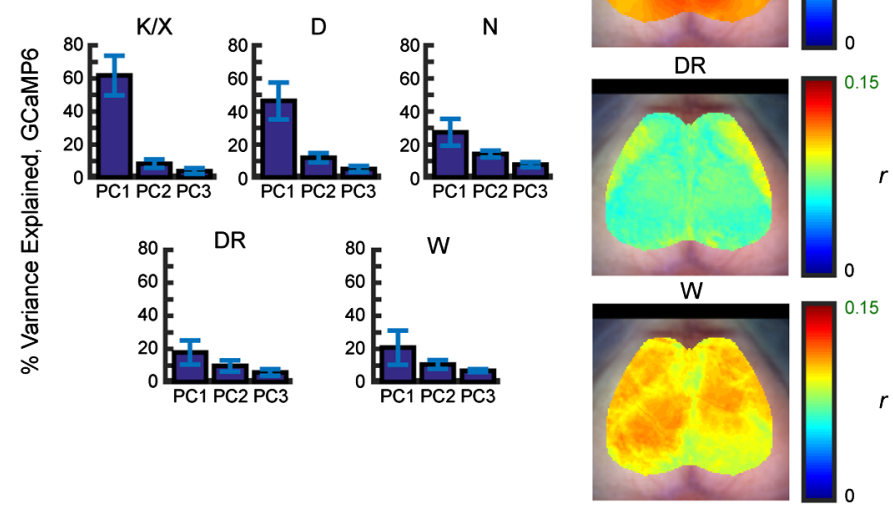

Lag Shifted GCaMP6/EEG Corr

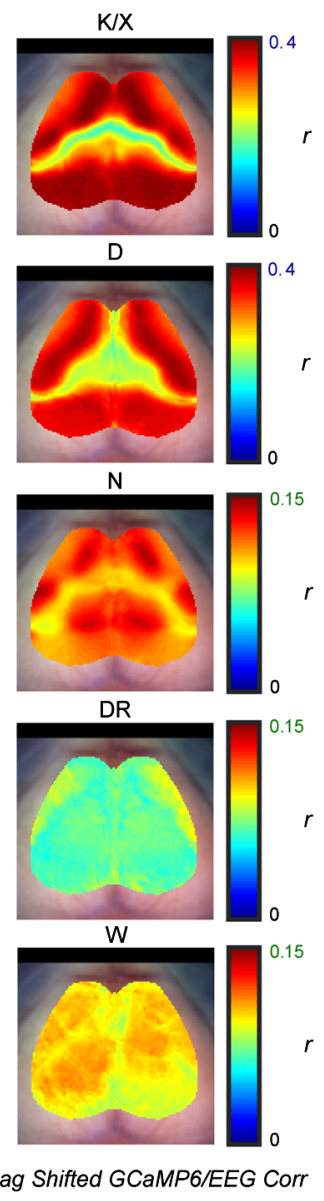

Fig. 4 The slow oscillation confounds correlation analysis in sleep and anesthesia but can be resolved into three PCs in delta GCaMP6 data. (a) Zero-lag correlation maps for ketamine/xylazine (K/X), $\operatorname{dex}(\mathrm{D})$, NREM (N), dex + reversal (DR), and wake (W) states averaged across all mice $(n=5)$. (b) Representations of the first three PCs for each brain state averaged across all mice $(n=5)$. (c) The average $(n=5)$ max cross-correlation between the lag-shifted summed first three PCs and delta EEG trace. (d) The average $(n=5)$ percent variability in the data accounted for by the first three PCs in each brain state. Error bars are standard deviations of the mean. (e) FC analysis $(n=5)$ performed on the data in each brain state after the first three PCs had been removed.

between these summed PCs and the filtered delta EEG trace resulted in high correlation coefficients in the sleep/anesthesia data when corrected for the lag between the two signals [Fig. 4(c) and Supplementary Figure S4]. This suggests that these first three PCs have captured characteristic features of NREM sleep and anesthesia. The first three PCs accounted for $\sim 45 \%, 60 \%$, and $70 \%$ of the variance in the sleep, dex, and ketamine/xylazine states, respectively, but only $35 \%$ and $34 \%$, respectively, in the wake and dex reversal states [Fig. 4(d)]. These observations combined suggested that the removal of the first three PCs should greatly reduce the variance attributable to the slow oscillation in the NREM/anesthesia data. The results of this maneuver are shown in Fig. 4(e), which illustrates a striking similarity of correlation structure across all brain states. Further, after removal of the first three PCs, all brain states are qualitatively similar to unmodified delta FC in the wake state [Fig. 4(a), lowest row; see also Fig. 8, Video 3, for similar spontaneous data across all brain states]. To quantify this observation, we evaluated the topographic similarity of FC maps computed in the PC 1-3 removed data compared to unmodified delta wake [Fig. 5(a), middle]. FC features unique to ketamine/xylazine, dex, and NREM (blue) were greatly attenuated post-PC removal, with only minor loss of homotopic FC typical of wake (white) [Fig. 5(b)]. Prior to PC 1-3 removal, modulated state specific FC topography differed by as much as $40 \%$ across states. Post-PC 1-3 removal, modulated state specific FC topography decreased over fourfold and became more uniform and similar to unmodified wake. These results suggest that the slow oscillation is superimposed onto canonical wake delta FC.

\section{Discussion}

We studied wake, NREM sleep, and anesthesia in transgenic mice expressing a genetically encoded calcium indicator using seed-based zero-lag correlation analysis (Figs. 3 and 4) and found brain state-, calcium/hemoglobin-, and frequency-dependent changes in FC structure. Specifically, within the infraslow frequency range, spontaneous hemoglobin activity patterns were roughly maintained across all brain states while neural activity patterns were slightly more varied. Neural activity within the delta range during sleep and anesthesia was dominated by the slow oscillation propagating in an anterior-to-posterior direction (Fig. 6, Video 1, Supplementary Figure S3A). Thus, 

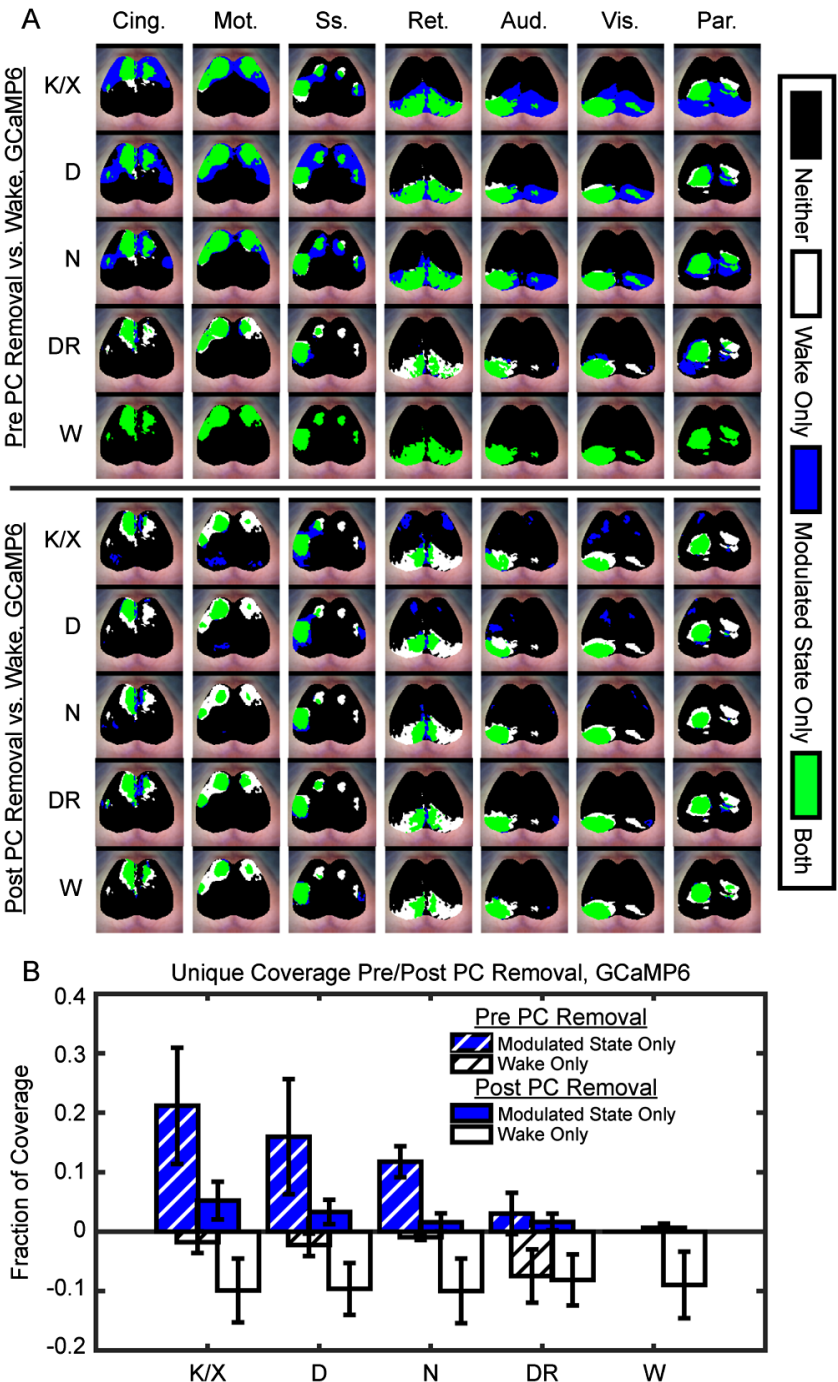

Fig. 5 Random effects analysis shows the return of wake-like FC patterns post-PC removal in delta GCaMP6 data. (a) Binary maps representing statistically significant functional correlations (random effects analysis, $n=5$ ) pre- and post-PC removal overlaid between wake and ketamine/xylazine $(K / X)$, dex (D), NREM $(N)$, dex+reversal (DR), or wake (W) (b) Quantification of brain coverage by unique functional correlations due to ketamine/xylazine, dex/dex+reversal, or NREM (blue), or wake (white) pre- and post-PC removal. Error bars are standard deviations across seeds.

we observed greater differences in correlation structure across brain states here. Finally, correlation structures that are qualitatively similar to wake delta FC patterns were recovered after linear decomposition removed the slow oscillation from delta neural activity data.

Previous FC studies of sleep and anesthesia have been conducted mostly in humans using fMRI, which effectively is restricted to temporal frequencies in the infraslow range $(0.009$ $0.08 \mathrm{~Hz}){ }^{45,46}$ Above $\sim 0.2 \mathrm{~Hz}$, hemodynamic signal power becomes essentially flat, which corresponds to a noise floor. ${ }^{50}$ Specifically, power in the oxygenated hemoglobin $\left(\mathrm{HbO}_{2}\right)$ signal follows a $1 / f$ spectral characteristic at infraslow frequencies, with minor changes in power across brain states. However, above infraslow frequencies, power across all brain states begins to be impacted by the aforementioned noise floor. This view is further supported by the present lag analysis performed on the delta-band hemoglobin data, which generated a topography dominated by vascular features (Supplementary Figure S3B). Nevertheless, our $\mathrm{HbO}_{2}$ infraslow correlation data showed modest brain state-dependent changes consistent with prior reports. ${ }^{18,20,21,51}$ Changes in correlation structure were slightly greater in the GCaMP6 infraslow data across brain state (e.g., the larger amplitude of ketamine/xylazine specific FC, Fig. 3(a), bottom). These results could reflect the faster dynamics provided by GCaMP6 fluorescence compared to $\mathrm{HbO}_{2}$ imaging.

In addition to infraslow dynamics, calcium imaging provides useful information at delta range frequencies, ${ }^{14}$ including the ability to study the sleep- and anesthesia-associated $\sim 1 \mathrm{~Hz}$ slow oscillation. It is this phenomenon that seems to drive the different correlation maps across brain state in the delta band. The strong nearly binary correlation/anti-correlation pattern seen in the sleep/anesthesia data visually captures a traveling wave, a defining feature of the slow oscillation. ${ }^{47}$ Notably, the difference between anesthesia and wake [Fig. 5(b)] is greater than the difference between NREM and wake, potentially suggesting that NREM architecture is "intermediate" to the anesthesia and wake architecture.

The present work is more comprehensive than previous similar studies in that we included sleep without anesthesia, more specifically NREM, and included two commonly used anesthetics - dexmedetomidine and ketamine/xylazine-in addition to baseline wake data. These comprehensive experimental conditions provide a foundation to evaluate the interaction between the slow oscillation and FC. Spontaneous neural activity often exhibits a " $1 / f$ " (scale-free) spectral characteristic. ${ }^{50,52}$ However, as shown by others, ${ }^{13,23,53}$ and again here, there may actually exist two regimes in the power spectral densities of spontaneous EEG as well as calcium signals under anesthesia or during NREM (see Fig. 2). The notion of superimposed spontaneous neural activity has been used before to describe " $1 / f$ like" versus oscillatory processes. ${ }^{52}$ It is plausible then that the removal of the sharp discontinuity of the $1 / f$ spectral characteristic in ketamine/xylazine, and the broader $1 / f$ discontinuity in the dex and NREM data is representative of a superimposed oscillatory event. Applying PCA to the NREM/anesthesia data, we observed that the slow oscillation was largely confined to the first three PCs. This was further reinforced by the high correlation between the delta EEG trace and the pixelwise isolated PCs, as well as the striking similarity between the delta GCaMP6 lag analysis topography [Fig. 2(c)] and the lag structures generated from the delta EEG and GCaMP6 PCA crosscorrelation analysis (Supplementary Figure S4). The increased GCaMP6 PCA/EEG correlation in the anesthesia and sleep brain states [Fig. 4(c)] relative to wake could be explained by global events driving the synchrony between the two manifestations of the slow oscillation. It should be further noted that discrepancies between the GCaMP6 and EEG data are to be expected, as EEG reports widely synchronous field potentials, whereas calcium imaging is more directly related to neural firing. Building on the observation that the first three PCs encompassed the slow oscillation, we demonstrated that wake-like delta spontaneous activity (as quantified through seed-correlation maps) could be recovered, and sharp spectral increases could be removed (Supplementary Figure S5A) from slow oscillation-dominated data by the removal of the first three PCs. Further, following delta band-pass filtering, GCaMP6 time series following PC $1-3$ removal are very similar to that 
recorded during wake (Supplementary Figure S5B,C, shaded panels). These results indicate that "wake-like" cortical activity persists during brain states dominated by the slow oscillation (e.g., NREM sleep and anesthesia) regardless of the mechanism of slow oscillation induction. Thus, during these brain states, the slow oscillation superimposes on, rather than replaces, persistent wake-like ongoing activity, the correlation structure of which is largely determined by anatomical connectivity. ${ }^{54-56}$

Ketamine/xylazine and dexmedetomidine induce stereotypical, invariant instantiations of the slow oscillation. ${ }^{31,57}$ Ketamine is an NMDA receptor antagonist, whereas xylazine and dex activate alpha-2 adrenergic receptors. Discrepant findings across the different forms of anesthesia could be due in part to the $40 \times$ increase in sedative efficacy of dex compared to xylazine, ${ }^{34}$ but much more likely, the antagonism of NMDA receptors and the downstream fine-tuning of excitation and inhibition lead to prolonged down states. ${ }^{17}$ This could explain the similar spatiotemporal properties of the slow oscillations induced by ketamine/xylazine and dex (Fig. 2) and the higher frequency at which dex-induced slow oscillations propagate [Fig. 2(a)]. By comparison, the calcium activity during NREM sleep was much more varied (Fig. 6, Video 1) than during anesthesia states. This variability may be attributable to variability in slow oscillation wave origin, amplitude, or direction of propagation. ${ }^{58}$ Alternatively, the complexity of the calcium dynamics during NREM relative to anesthesia may result from a mixing of lower amplitude slow oscillations with relatively higher amplitude spontaneous wake-like activity. Even during wake, the first two PCs included features suggestive of the slow oscillation [Figs. 4(b) and 7, Video 2], albeit in greatly attenuated magnitude and contributing to less variance. This result potentially reflects microsleep ${ }^{59,60}$ or brief periods of reduced alertness within the 10-s EEG epochs used to score the optical data. ${ }^{61}$

\section{Caveats, Future Directions, and Conclusions}

Sleep deprivation over $6 \mathrm{~h}$ prior to calcium (GCaMP6) imaging was used to increase the quantity of NREM sleep. This is a potential caveat as sleep deprivation has been reported to change the shape, incidence, and amplitude of slow oscillations. ${ }^{62}$ It remains to be determined whether wide-field image acquisition of freely behaving natural sleep is possible for extended durations and if differences between nonsleep-deprived and sleep-deprived sleep exist. However, this issue presumably does not impact the present anesthesia results.

On the other hand, it has been reported that slow wave activity reflects sleep pressure, i.e., that the prevalence of slow waves at the start of NREM is proportional to prior wake time. ${ }^{63}$ Although the present study was not designed to examine this effect, it is possible that reduced power in the dex reversal data, ${ }^{31}$ as compared to normal wake [Figs. 2(a), 2(b), and Supplementary Figure S2], reflects reduced sleep pressure following $30 \mathrm{~min}$ of anesthesia-induced slow oscillations. Future experiments controlling for time spent asleep versus awake in sleep-deprived and nonsleep-deprived brain states could rigorously address this question.

Finally, while algorithms more sophisticated than PCA may provide improved removal of the slow oscillation, PCA works sufficiently to establish this concept. As expected with PCA, the first three PCs may include some variance not specifically tied to the slow oscillation. Further, the match between Fig. 4(e) and wake topography [lowest row of Fig. 4(a)] is not perfect. However, the match is sufficient to demonstrate our principal claim that after removing the slow oscillation, the remaining signals reflect patterns [Fig. 4(e)] expected of FC in the wake state [Fig. 4(a), bottom row] and, importantly, that these features do not suggest artifact. Recovery of focal FC patterns post-linear decomposition supports the idea that the slow oscillation superimposes on wake-like intrinsic activity.

Prior studies have made inferences about changes in connectivity across experimental brain states or under the global synchronization of anesthesia. ${ }^{15,64,65}$ In the future, it will thus be important to closely monitor the brain state of the animal when conducting FC analysis. The present study presents promising avenues of future study to focus on the transition between sleep and wake or the specific cortical activity underlying sleep. More broadly, this work opens up avenues of research that may elucidate underlying mechanisms of sleep and sleep disturbances consequent to disease. For example, our findings may have implications for understanding how sleep and Alzheimer's disease interact ${ }^{66,67}$ or how anesthesia influences postoperative delirium. ${ }^{68}$

\section{Appendix A: Real-Time Data}

\subsection{Spontaneous Activity During Anesthesia, NREM, and Wake}

Data was processed as described above and visualized for the five experimental brain states included in the present study (Fig. 6).

\subsection{Slow Oscillation Isolation using Linear Decomposition}

Summation of the first three principal components isolated the slow oscillation spatio-temporal feature present during NREM sleep and the forms of anesthesia used in the present study (Fig. 7).
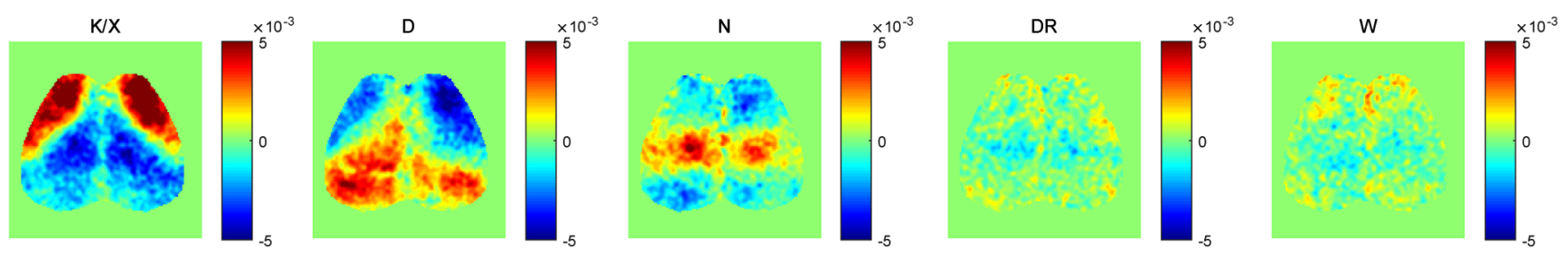

Fig. 6 Example GCaMP6 data in real time for each brain state. $20 \mathrm{~s}$ in real time of example GCaMP6 data $(\Delta F / F)$ from one mouse for each brain state: ketamine/xylazine $(K / X)$, dex $(D)$, NREM $(N)$, dex + reversal (DR), and wake (W). Videos were smoothed with a $2 \times 2$ Gaussian filter (Video 1, MPEG-4, 18.3 MB [URL: https://doi.org/10.1117/1.NPh.6.3.035002.1]). 

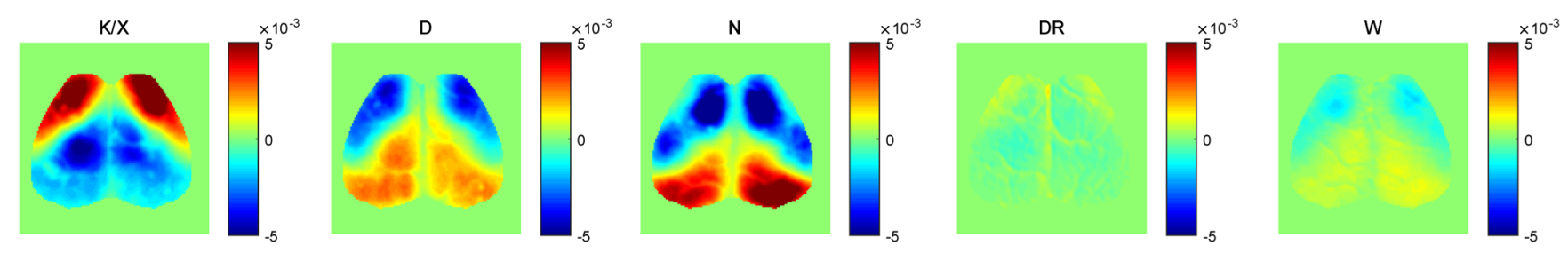

Fig. 7 Example PC analysis data in real time for each brain state. $20 \mathrm{~s}$ in real time of GCaMP6 data $(\Delta F / F)$ from one mouse using only the summed first three PCs for each brain state: ketamine/xylazine $(K / X)$, dex (D), NREM (N), dex + reversal (DR), and wake (W). Videos were smoothed with a $2 \times 2$ Gaussian filter (Video 2, MPEG-4, 10.8 MB [URL: https://doi.org/10.1117/1.NPh.6.3.035002.2]).
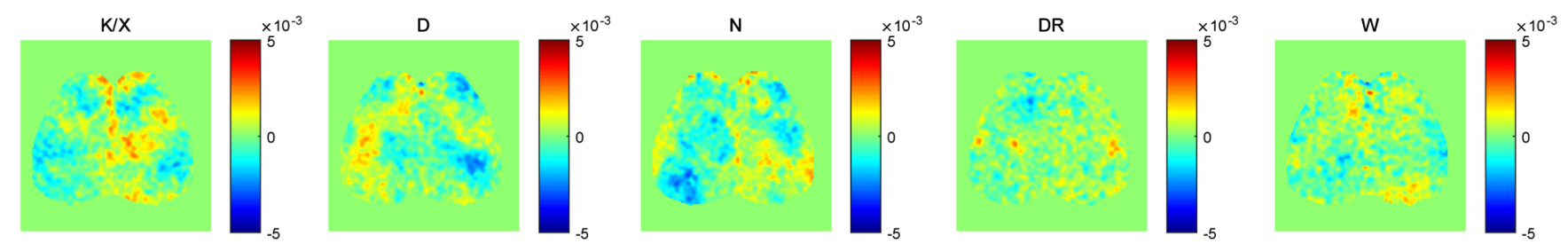

Fig. 8 Example GCaMP6 data in real time after removal of the first three PCs. $20 \mathrm{~s}$ in real time of GCaMP6 data $(\Delta F / F)$ from one mouse after PCs 1-3 were removed from each brain state: ketamine/ xylazine $(K / X)$, dex (D), NREM (N), dex + reversal (DR), and wake (W). Videos were smoothed with a $2 \times 2$ Gaussian filter (Video 3, MPEG-4, 17.1 MB [URL: https://doi.org/10.1117/1.NPh.6.3.035002.3]).

\subsection{Spontaneous Activity Underlying the Slow Oscillation}

Spontaneous activity remaining in the data after removal of the spatio-temporal feature isolated in the first three principal components (Fig. 8).

\section{Disclosures}

The authors have no conflicts of interest to disclose.

\section{Acknowledgments}

We are grateful to Armand Mensen for his insightful review, edits, and analysis suggestions. This work was supported by the National Institute of Neurological Disorders and Stroke [Grant Nos. R01NS078223 (to J.P.C.), R01NS084028 and R37NS110699 (to J.M.L.), R01NS102870 (to A.Q.B.), K25NS083754 (to A.Q.B.), and P01NS080675 (to J.P.C. and A.Z.S.)]; the American Academy of Sleep Medicine Foundation [Grant No. 183-PA-18 (to E.C.L.)]; and the Eunice Kennedy Shriver National Institute of Child Health and Human Development [Grant No. U54HD087011 (to J.P.C.)]. Sample data and/or code for the supplementary data will be made available upon request.

\section{References}

1. B. B. Biswal et al., "Toward discovery science of human brain function," Proc. Natl. Acad. Sci. U. S. A. 107, 4734-4739 (2010).

2. M. E. Raichle, "The restless brain: how intrinsic activity organizes brain function," Philos. Trans. R. Soc. Lond. B Biol. Sci. 370, 20140172 (2015).

3. S. M. Smith et al., "Functional connectomics from resting-state fMRI," Trends Cognit. Sci. 17, 666-682 (2013).

4. D. S. Bassett and O. Sporns, "Network neuroscience," Nat. Neurosci. 20, 353-364 (2017).

5. L. G. Apostolova and P. M. Thompson, "Brain mapping as a tool to study neurodegeneration," Neurotherapeutics 4, 387-400 (2007).
6. J. S. Anderson et al., "Reproducibility of single-subject functional connectivity measurements," Am. J. Neuroradiol. 32, 548-555 (2011).

7. S. W. Hughes et al., "Infraslow $(<0.1 \mathrm{~Hz})$ oscillations in thalamic relay nuclei basic mechanisms and significance to health and disease states," Prog. Brain Res. 193, 145-162 (2011).

8. B. Biswal et al., "Functional connectivity in the motor cortex of resting human brain using echo-planar MRI," Magn. Reson. Med. 34, 537-541 (1995).

9. A. T. Eggebrecht et al., "A quantitative spatial comparison of highdensity diffuse optical tomography and fMRI cortical mapping," Neurolmage 61, 1120-1128 (2012).

10. B. R. White et al., "Imaging of functional connectivity in the mouse brain," PLoS One 6, e16322 (2011).

11. Y. Ma et al., "Wide-field optical mapping of neural activity and brain haemodynamics: considerations and novel approaches," Philos. Trans. R. Soc. Lond. B Biol. Sci. 371, 20150360 (2016).

12. M. P. Vanni and T. H. Murphy, "Mesoscale transcranial spontaneous activity mapping in GCaMP3 transgenic mice reveals extensive reciprocal connections between areas of somatomotor cortex," J. Neurosci. 34, 15931-15946 (2014).

13. P. W. Wright et al., "Functional connectivity structure of cortical calcium dynamics in anesthetized and awake mice," PLoS One. 12, e0185759 (2017).

14. L. Tian et al., "Imaging neural activity in worms, flies and mice with improved GCaMP calcium indicators," Nat. Methods 6, 875-881 (2009).

15. M. Steriade, A. Nunez, and F. Amzica, "A novel slow $(<1 \mathrm{~Hz})$ oscillation of neocortical neurons in vivo: depolarizing and hyperpolarizing components," J. Neurosci. 13, 3252-3265 (1993).

16. G. T. Neske, "The slow oscillation in cortical and thalamic networks: mechanisms and functions," Front. Neural Circuits 9, 88 (2016).

17. S. Chauvette et al., "Properties of slow oscillation during slow-wave sleep and anesthesia in cats," J. Neurosci. 31, 14998-15008 (2011).

18. L. J. Larson-Prior et al., "Cortical network functional connectivity in the descent to sleep," Proc. Natl. Acad. Sci. 106, 4489-4494 (2009).

19. D. Picchioni, J. H. Duyn, and S. G. Horovitz, "Sleep and the functional connectome," Neuroimage 80, 387-396 (2013).

20. P. G. Samann et al., "Development of the brain's default mode network from wakefulness to slow wave sleep," Cereb. Cortex 21, 2082-2093 (2011).

21. E. Tagliazucchi and H. Laufs, "Decoding wakefulness levels from typical fMRI resting-state data reveals reliable drifts between wakefulness and sleep," Neuron 82, 695-708 (2014). 
22. R. N. Mhuircheartaigh et al., "Cortical and subcortical connectivity changes during decreasing levels of consciousness in humans: a functional magnetic resonance imaging study using propofol," J. Neurosci. 30, 9095-9102 (2010).

23. A. W. Chan et al., "Mesoscale infraslow spontaneous membrane potential fluctuations recapitulate high-frequency activity cortical motifs," Nat. Commun. 6, 7738 (2015).

24. I. Ferezou, S. Bolea, and C. C. H. Petersen, "Visualizing the cortical representation of whisker touch: voltage-sensitive dye imaging in freely moving mice," Neuron 50, 617-629 (2006).

25. M. H. Mohajerani et al., "Mirrored bilateral slow-wave cortical activity within local circuits revealed by fast bihemispheric voltage-sensitive dye imaging in anesthetized and awake mice," J. Neurosci. 30, 3745-3751 (2010).

26. G. Silasi et al., "Intact skull chronic windows for mesoscopic wide-field imaging in awake mice," J. Neurosci. Methods 267, 141-149 (2016).

27. J. Sleigh et al., "Ketamine- More mechanisms of action than just NMDA blockade," Trends Anaesth. Crit. Care 4, 76-81 (2014).

28. R. Gertler et al., "Dexmedetomidine: a novel sedative-analgesic agent," Proc. (Bayl. Univ. Med. Cent.) 14, 13-21 (2001).

29. S. Buitrago et al., "Safety and efficacy of various combinations of injectable anesthetics in BALB/c mice," J. Am. Assoc. Lab. Anim. Sci. 47(1), 11-17 (2008).

30. C. Correa-Sales, B. C. Rabin, and M. Maze, "A hypnotic response to dexmedetomidine, an $\alpha 2$ agonist, is mediated in the locus coeruleus in rats," Anesthesiology 1992, 76(6), 948-952.

31. E. Nelson Laura et al., "The $\alpha 2$-adrenoceptor agonist dexmedetomidine converges on an endogenous sleep-promoting pathway to exert its sedative effects," Anesthesiology 98, 428-436 (2003).

32. O. Akeju et al., "Electroencephalogram signatures of ketamine anesthesia-induced unconsciousness," Clin. Neurophysiol. 127, 2414-2422 (2016).

33. M. B. Struck et al., "Effect of a short-term fast on ketamine-xylazine anesthesia in rats," J. Am. Assoc. Lab. Anim. Sci. 50(3), 344-348 (2011).

34. D. Wellington, I. Mikaelian, and L. Singer, "Comparison of ketaminexylazine and ketamine-dexmedetomidine anesthesia and intraperitoneal tolerance in rats," J. Am. Assoc. Lab. Anim. Sci. 52(4), 481-487 (2013).

35. I. Tobler and A. A. Borbély, "The effect of 3-h and 6-h sleep deprivation on sleep and EEG spectra of the rat," Behav. Brain Res. 36, 73-78 (1990).

36. Y. Oishi et al., "Polygraphic recording procedure for measuring sleep in mice," J. Vis. Exp. 107, e53678 (2016).

37. Y. Ma et al., "Resting-state hemodynamics are spatiotemporally coupled to synchronized and symmetric neural activity in excitatory neurons," Proc. Natl. Acad. Sci. U. S. A. 113, E8463-E8471 (2016).

38. M. D. Fox and M. E. Raichle, "Spontaneous fluctuations in brain activity observed with functional magnetic resonance imaging," Nat. Rev. Neurosci. 8, 700-711 (2007).

39. T. T. Dang-Vu et al., "Cerebral correlates of delta waves during nonREM sleep revisited," Neuroimage 28, 14-21 (2005).

40. K. J. Friston, A. P. Holmes, and K. J. Worsley, "How many subjects constitute a study?" NeuroImage 10, 1-5 (1999).

41. D. Xiao et al., "Mapping cortical mesoscopic networks of single spiking cortical or sub-cortical neurons," Elife 6, e19976 (2017).

42. O. Akeju et al., "Spatiotemporal dynamics of dexmedetomidineinduced electroencephalogram oscillations," PLoS One 11, e0163431 (2016).

43. F. Amzica and M. Steriade, "Electrophysiological correlates of sleep delta waves," Electroencephalogr. Clin. Neurophysiol. 107, 69-83 (1998).

44. M. D. H. Scheinin et al., "Reversal of the sedative and sympatholytic effects of dexmedetomidine with a specific $\alpha 2$-adrenoceptor antagonist atipamezolea pharmacodynamic and kinetic study in healthy volunteers," Anesthesiology 89, 574-584 (1998).

45. J. S. Anderson, "Origin of synchronized low-frequency blood oxygen level-dependent fluctuations in the primary visual cortex," Am. J. Neuroradiol. 29, 1722-1729 (2008).

46. G. M. Hathout et al., "The lag of cerebral hemodynamics with rapidly alternating periodic stimulation: modeling for functional MRI," Magn. Reson. Imaging 17, 9-20 (1999).

47. M. Massimini et al., "The sleep slow oscillation as a traveling wave," J. Neurosci. 24, 6862-6870 (2004).
48. N. Niethard et al., "Cortical circuit activity underlying sleep slow oscillations and spindles," Proc. Natl. Acad. Sci. 115(39), E9220-E9229 (2018).

49. T. Hiltunen et al., "Infra-slow EEG fluctuations are correlated with resting-state network dynamics in fMRI," J. Neurosci. 34, 356-362 (2014).

50. B. J. He, "Scale-free brain activity: past, present, and future," Trends Cognit. Sci. 18, 480-487 (2014).

51. S. G. Horovitz et al., "Decoupling of the brain's default mode network during deep sleep," Proc. Natl. Acad. Sci. U. S. A. 106, 11376-11381 (2009).

52. W. J. Freeman et al., "Spatial spectral analysis of human electrocorticograms including the alpha and gamma bands," J. Neurosci. Methods 95, 111-121 (2000).

53. A. Mitra et al., "Human cortical-hippocampal dialogue in wake and slow-wave sleep," Proc. Natl. Acad. Sci. 113, E6868-E6876 (2016).

54. S. W. Oh et al., "A mesoscale connectome of the mouse brain," Nature 508, 207-214 (2014).

55. C. J. Honey et al., "Predicting human resting-state functional connectivity from structural connectivity," Proc. Natl. Acad. Sci. U. S. A. 106, 2035-2040 (2009).

56. H. Johansen-Berg, "Human connectomics-what will the future demand?" Neuroimage 80, 541-544 (2013).

57. M. T. Alkire and J. Miller, "General anesthesia and the neural correlates of consciousness," Prog. Brain Res. 150, 229-244 (2005).

58. D. Shimaoka, C Song, and T. Knöpfel, "State-dependent modulation of slow wave motifs towards awakening," Front. Cell. Neurosci. 11, 108 (2017).

59. Y. Harrison and J. A. Horne, "Occurrence of 'microsleeps' during day time sleep onset in normal subjects," Electroencephalogr. Clin. Neurophysiol. 98(5), 411-416 (1996).

60. U. Hemmeter et al., "Microsleep during partial sleep deprivation in depression," Biol. Psychiatr. 43, 829-839 (1998).

61. V. V. Vyazovskiy et al., "Local sleep in awake rats," Nature 472, 443447 (2011).

62. V. V. Vyazovskiy et al., "Cortical firing and sleep homeostasis," Neuron 63, 865-878 (2009).

63. A. A. Borb and P. Achermann, "Sleep homeostasis and models of sleep regulation," J. Biol. Rhythms. 14, 559-570 (1999).

64. M. Boly et al., "Connectivity changes underlying spectral EEG changes during propofol-induced loss of consciousness," J. Neurosci. 32, 70827090 (2012).

65. M. Steriade, I. Timofeev, and F. Grenier, "Natural waking and sleep states: a view from inside neocortical neurons," J. Neurophysiol. 85, 1969-1985 (2001).

66. M. M. Lim, J. R. Gerstner, and D. M. Holtzman, "The sleep-wake cycle and Alzheimer's disease: what do we know?" Neurodegener. Dis. Manage. 4, 351-362 (2014).

67. Y.-E. S. Ju, B. P. Lucey, and D. M. Holtzman, "Sleep and Alzheimer disease pathology—a bidirectional relationship," Nat. Rev. Neurol. 10, 115-119 (2013).

68. J. M. Leung et al., "Preoperative sleep disruption and postoperative delirium," J. Clin. Sleep Med. 11, 907-913 (2015).

Lindsey $\mathbf{M}$. Brier is currently an $\mathrm{MD} / \mathrm{PhD}$ candidate in the Neurosciences Program at Washington University in St. Louis. She works in Dr. Joseph Culver's lab designing single photon wide-field illumination systems in order to answer neurological questions. Previously, she received a BS degree in mathematical biology from the University of Houston in 2014.

Eric C. Landsness is an instructor in the Department of Neurology at Washington University in St. Louis. He received his electrical engineering degree at the University of Washington in 2003, MD and $\mathrm{PhD}$ degrees from the University of Wisconsin, Madison, in 2013, and completed a neurology residency and sleep fellowship at Washington University, St. Louis, in 2018. He is interested in the role of sleep and stroke recovery.

Abraham Z. Snyder received an $A B$ degree in chemistry from Columbia University in New York, in 1970, PhD in neurophysiology from Rockefeller University in 1977, and MD from the State University of New York at Buffalo in 1981. He is a research professor of radiology and research associate professor of neurology at 
Washington University in St. Louis. His primary research interest is intrinsic brain activity and resting-state FC.

Patrick W. Wright is currently a postdoctoral research fellow under Alan Koretsky, PhD, in the Laboratory of Functional and Molecular Imaging at the National Institute of Neurological Disorders and Stroke, U.S. National Institutes of Health. He received his BS degree in biomedical engineering from the University of Alabama at Birmingham in 2010. He subsequently earned his $\mathrm{PhD}$ in biomedical engineering from Washington University in St. Louis in December 2016 with Joseph Culver, PhD, and Beau Ances, MD, PhD, serving as his thesis advisers. During graduate school, he developed an in vivo optical imaging system to study resting-state cortical activity using concurrently-measured hemodynamics and fluorescent calcium transients in mice.

Grant A. Baxter was a research assistant at Washington University in St. Louis in the Department of Radiology under the guidance of Dr. Joseph Culver.

Adam Q. Bauer is currently an assistant professor of radiology and biomedical engineering at Washington University in St. Louis. His lab develops functional optical imaging methods for translational neuroscience applications in rodents. He received his BS degree in physics at Truman State University in 2001 and his PhD in physics from Washington University in St. Louis in 2009 in the Laboratory of Dr. James G. Miller.

Jin-Moo Lee is the Norman J. Stupp Professor of Neurology at Washington University School of Medicine. He received his BA degree at Yale College, MD and $\mathrm{PhD}$ degrees in neuroscience at Weill Cornell Medical College. His research focuses on stroke and brain plasticity and recovery after stroke.

Joseph P. Culver received his BS degree in physics from the University of Washington in 1988 and his $\mathrm{PhD}$ in physics from the University of Pennsylvania in 1997 under Drs. Arjun G. Yodh and Robin M. Hochstrasser. He is the Sherwood Moore Professor of Radiology and a professor of physics and biomedical engineering at Washington University in St. Louis. His lab explores ways of exploiting noninvasive optical measurements for both functional and molecular biological imaging. 